\title{
MELHORES PRÁTICAS NO CUIDADO À PESSOA COM DOENÇA CARDIOVASCULAR: INTERFACE ENTRE LIDERANÇA E SEGURANÇA DO PACIENTE*
}

\author{
Giuliana Caldeirini Aruto ${ }^{1}$, Gabriela Marcellino de Melo Lanzoni ${ }^{2}$, Betina Hörner Schlindwein Meirelles ${ }^{2}$
}

\begin{abstract}
RESUMO: Estudo descritivo, com abordagem qualitativa, que objetivou conhecer as melhores práticas do enfermeiro, bem como propor aprimoramentos no cuidado seguro à pessoa com doença cardiovascular atendida em instituição hospitalar privada especializada em cardiologia no Sul do Brasil. Desenvolvido entre agosto e outubro de 2015, nas três Unidades de Clínica Cirúrgica, com participação de 14 enfermeiros. Coleta de dados realizada através de entrevista semiestruturada e utilizou-se como referencial a Análise de Conteúdo. Foram identificadas melhores práticas nas ações de cuidado ao paciente, melhores práticas de liderança do enfermeiro, e por fim, proposições para o aprimoramento das melhores práticas para o cuidado seguro à pessoa com doença cardiovascular. Há necessidade de investir na cultura de segurança, no aperfeiçoamento da equipe de enfermagem, na utilização de melhores práticas e melhoria dos ambientes de trabalho para o alcance da segurança do paciente.
\end{abstract}

DESCRITORES: Enfermagem cardiovascular; Cuidados de enfermagem; Liderança; Gerenciamento clínico; Segurança do paciente.

\section{BEST PRACTICES IN CARE FOR PEOPLE WITH CARDIOVASCULAR DISEASES: THE INTERFACE BETWEEN LEADERSHIP AND PATIENT SAFETY}

\begin{abstract}
Descriptive study with a qualitative approach. The objective was to identify best nursing practices and propose improvements in safe care of patients with cardiovascular diseases in a private hospital specializing in cardiology in southern Brazil. The study was conducted from August to October 2015 in the hospital's 3 surgery units; 14 nurses participated. The data collection was performed with semi-structured interviews using content analysis as a framework. Best practices in patient safety and nursing leadership were identified, as well as proposals for improvements in those practices to ensure safe care of patients with cardiovascular diseases. The results showed a need to invest in patient safety culture, nursing team improvements, use of best practices, and upgraded work environments to enhance patient safety.
\end{abstract}

DESCRIPTORS: Cardiovascular nursing; Nursing care; Leadership; Disease management; Patient safety.

\section{MEJORES PRÁCTICAS DE CUIDADO A PERSONA CON ENFERMEDAD CARDIOVASCULAR: INTERFAZ LIDERAZGO / SEGURIDAD DEL PACIENTE}

RESUMEN: Estudio descriptivo de abordaje cualitativo, con fines de conocer las mejores prácticas del enfermero, así como proponer mejoras en el cuidado seguro a la persona con enfermedad cardiovascular atendida en institución hospitalaria privada especializada en cardiología del Sur de Brasil. Desarrollado entre agosto y octubre de 2015, en las tres Unidades de Clínica Quirúrgica, con participación de 14 enfermeros. Recolección realizada mediante entrevista semiestructurada, utilizándose como referencia el Análisis de Contenido. Fueron identificadas mejores prácticas en las acciones de cuidado al paciente, mejores prácticas de liderazgo del enfermero $y$, finalmente, propuestas de mejoramiento de las mejores prácticas para el cuidado seguro de la persona con enfermedad cardiovascular. Existe necesidad de invertir en la cultura de seguridad, en la mejora de los ámbitos laborales del equipo de enfermería, en la aplicación de mejores prácticas y mejoramiento de los ambientes laborales para el alcance de la seguridad del paciente.

DESCRIPTORES: Enfermería cardiovascular; Atención de enfermería; Liderazgo; Manejo de la enfermedad; Seguridad del paciente.

*Artigo extraído da Dissertação de Mestrado intitulada: "Melhores práticas no cuidado de enfermagem seguro às pessoas com doenças cardiovasculares: subsídios para liderança". Universidade Federal de Santa Catarina, 2016.

${ }^{1}$ Enfermeira. Mestre em Enfermagem. Universidade Federal de Santa Catarina. Florianópolis, SC, Brasil.

${ }^{2}$ Enfermeira. Doutora em Enfermagem. Docente de Enfermagem da Universidade Federal de Santa Catarina. Florianópolis, SC, Brasil.

Autor Correspondente:

Gabriela Marcellino de Melo Lanzoni

Universidade Federal de Santa Catarina

R. Delfino Conti, s/n - 88040-900 - Florianópolis, SC, Brasil

E-mail: gabimrc@gmail.com
Recebido: 01/03/2016

Finalizado: 01/07/2016 


\section{INTRODUÇÃO}

O cuidado prestado às pessoas com doenças cardiovasculares é complexo e requer que seja executado com qualidade e sem gerar danos desnecessários ao indivíduo. Apenas enfermeiros que conhecem as melhores práticas relativas ao cuidado cardiovascular podem garantir um resultado excelente a essas pessoas ${ }^{(1)}$.

O termo "melhores práticas" refere-se às práticas de enfermagem que são baseadas na melhor evidência disponível a partir de pesquisas em enfermagem. Seu objetivo é aplicar as intervenções de enfermagem mais recentes, relevantes e úteis, baseadas nas pesquisas e nas práticas diárias ${ }^{(2)}$.

O entendimento a respeito das melhores práticas pode possibilitar aos enfermeiros um pensar e agir mais efetivo, dando subsídios para sistematizar sua prática de trabalho, tornando-a clara, definida e com legibilidade, para garantir uma prática segura com consequente qualidade e satisfação do paciente ${ }^{(3)}$. Por estarem constantemente prestando cuidado direto ao paciente, os enfermeiros, juntamente com a equipe de enfermagem, são os elementos chave no processo de evitar erros, impedir decisões ruins e também de assumir um papel de liderança no avanço e no uso de estratégias para promover a qualidade do cuidado e segurança do paciente ${ }^{(4)}$.

A expressão "segurança do paciente" faz referência aos fatores que influenciam as instituições a empregar a cultura de segurança, levando-se em consideração as melhores práticas ${ }^{(5)}$. Nas instituições de saúde, as pessoas com doença cardiovascular contam com os cuidados do profissional enfermeiro e da equipe de enfermagem nas mais diversas ordens, desde os atendimentos de emergências até os procedimentos invasivos. Esses cuidados muitas vezes podem gerar eventos adversos, os quais provêm de erros ou falhas na realização dos cuidados de enfermagem ou nos processos assistenciais, podendo colocar em risco a segurança do paciente.

Neste contexto, o Ministério da Saúde em parceria com a Agência Nacional de Vigilância Sanitária (ANVISA) lançaram, a partir de 2013, a série "Segurança do Paciente e Qualidade em Serviços de Saúde", sendo composta por seis manuais e seis protocolos básicos na temática de segurança do paciente. Através destes manuais e protocolos, é possível a atualização do conhecimento com as melhores práticas apresentadas, a redução e a mitigação de riscos e atos inseguros, e a melhora da qualidade do cuidado prestado nas instituições de saúde ${ }^{(4)}$. Este estudo utilizou esses protocolos e manuais como referencial teórico, pois através destes é possível determinar quais são os cuidados considerados mais relevantes, atuais e que garantam qualidade e segurança ao paciente.

Diante do exposto, torna-se importante conhecer quais são as melhores práticas adotadas pelo enfermeiro e sua equipe no cuidado seguro à pessoa com doença cardiovascular. A adesão às melhores práticas acarreta melhor desempenho, reduzindo a morbimortalidade e o custo das doenças cardiovasculares, além de contribuir para maior segurança dos pacientes ${ }^{(6)}$.

Desse modo, esse estudo teve como objetivo conhecer as melhores práticas do enfermeiro, bem como propor aprimoramentos no cuidado seguro à pessoa com doença cardiovascular atendida em uma instituição hospitalar privada especializada em cardiologia no Sul do Brasil.

\section{MÉTODO}

Trata-se de um estudo descritivo, com abordagem qualitativa, desenvolvido em uma instituição hospitalar privada especializada em cardiologia no Sul do Brasil, no período entre agosto e outubro de 2015.

Os locais de pesquisa foram as três Unidades de Clínica Cirúrgica (UCC), sendo que a UCC1 conta com 19 leitos de internação, a UCC2 e UCC3 contam com 18 leitos de internação respectivamente.

A equipe de enfermagem na UCC1 e UCC3 constitui-se por 8 enfermeiros e 28 técnicos de enfermagem. A equipe de enfermagem na UCC 2 constitui-se por 8 enfermeiros e 8 técnicos de enfermagem, sendo que no período diurno a unidade apresenta 3 enfermeiros assistenciais realizando os cuidados integrais aos pacientes e 1 técnico de enfermagem para auxiliar os enfermeiros; já no 
período noturno ocorre o oposto, a equipe é composta por 1 enfermeiro e 3 técnicos de enfermagem. A escala de trabalho nas unidades é 12 horas de trabalho por 36 horas de descanso.

Como critério de inclusão considerou-se: ser enfermeiro que atue na unidade há no mínimo três meses. Os critérios de exclusão adotados foram: enfermeiros com menos de três meses de vínculo na mesma unidade de internação e estar de férias ou licença no período da coleta dos dados. Dos 16 enfermeiros em atividade, o estudo contou com a participação de 14 enfermeiros, já que 2 não atendiam ao critério de inclusão.

A coleta de dados foi realizada através de entrevista semiestruturada no próprio hospital. Esta era composta de questões norteadoras sobre segurança do paciente, melhores práticas para o cuidado seguro e prevenção de eventos adversos à pessoa com doença cardiovascular. As entrevistas foram gravadas em meio digital e os participantes foram entrevistados em um único encontro.

Após esta etapa, as entrevistas foram transcritas em sua íntegra, passaram por processo analítico a partir do referencial da Análise de Conteúdo. Tal referencial é composto de três fases: a pré-análise, a exploração do material e o tratamento dos resultados (a inferência e a interpretação) ${ }^{(7)}$.

A pré-análise inicia-se com a leitura de todos os dados por diversas vezes para obter o sentido do todo; esse primeiro contato com os dados se constitui no que Bardin chama de "leitura flutuante"(7). A segunda fase aborda a exploração do material ou codificação, que consiste na definição de categorias através da codificação(7). Destaca-se que as categorias não foram pré-estabelecidas, as categorizações foram feitas manualmente por agrupamento dos códigos que emergiram, a partir da similaridade e diferenças de idéias pautadas nas metas internacionais de segurança do paciente. O último momento consiste no tratamento dos resultados, quando ocorre a condensação e o destaque das informações para análise, propõe inferências e realiza interpretações ${ }^{(7)}$. Nesta última etapa emergiram da análise dos dados 3 categorias e 17 subcategorias.

Para garantir o anonimato dos participantes, estes foram identificados com as letras 'EA' para os enfermeiros assistenciais, e 'E' para os demais enfermeiros, seguindo de numeral arábico conforme a ordem em que foram entrevistados. A pesquisa foi aprovada pelo Comitê de Ética em Pesquisa com Seres Humanos da Secretaria de Estado da Saúde de Santa Catarina/SES sob protocolo n 1.049.447.

\section{RESULTADOS}

Os achados apontam a interface entre liderança do enfermeiro e a segurança do paciente na compreensão das melhores práticas de cuidado à pessoa com doença cardiovascular, os quais serão apresentados em três categorias temáticas: "Melhores práticas nas ações de cuidado ao paciente", "Melhores práticas de liderança do enfermeiro" e "Proposições para o aprimoramento das melhores práticas".

\section{Melhores práticas nas ações de cuidado ao paciente}

Essa categoria é composta por cinco subcategorias, que abordam as melhores práticas de cuidado na esfera das relações diretas entre profissional e paciente hospitalizado.

Na subcategoria "Conferência da identificação do paciente", verificou-se que esta é realizada através da pulseira de identificação e a sinalização do leito. Quando o paciente chega à unidade de internação, o enfermeiro confere o seu nome, juntamente com a pulseira e o prontuário. Para os enfermeiros, a pulseira e a identificação no leito proporcionaram melhora da comunicação entre enfermagem e o paciente, já que o seu nome fica visível a todos os profissionais, evitando dessa forma erros de identificação.

O paciente chega, a gente imprime o nome dele e coloca em cima do leito, e tem a pulseirinha com a identificação do paciente [...] Então, é bom chegar ao paciente e falar o nome certo, porque às vezes a gente não lembra né? Então, é bom, você olhar para cima e vê em cima do leito o nome. Isso melhorou a qualidade, melhorou a segurança, foi fundamental. (E3) 
Na subcategoria "Prevenção de infecção hospitalar", destacam-se elementos relevantes para a segurança do paciente. Para prevenir a infecção hospitalar, a lavagem das mãos é um cuidado primordial, presente em todos os momentos, e atrelado a este cuidado está a assepsia dos materiais.

Os enfermeiros relataram a prática do isolamento preventivo por precaução de contato. Quando existe a suspeita de o paciente estar infectado por bactéria multirresistente ou é proveniente de outra instituição, ocorre o isolamento preventivo. Nesses casos, o paciente fica preferencialmente sozinho no quarto, sendo necessário possuir os equipamentos de proteção individual próximo ao leito, juntamente com materiais para verificar sinais vitais e quartenário de amônia para higienizar os materiais e móveis. Para identificar que o paciente está em precaução de contato, é colocada a sinalização de "Precaução de contato" na porta, informando os cuidados necessários ao entrar no quarto.

Na subcategoria, "Conferência de medicamentos", todos os enfermeiros referiram esta prática como fundamental para a administração segura de medicamentos. É indicada a conferência desde a dispensação destes na farmácia com a dupla checagem até a administração ao paciente.

Quando há presença de pacientes com nomes iguais ou parecidos sob o cuidado do mesmo profissional, é referido pelos participantes que os enfermeiros redistribuem os profissionais da escala, para que o mesmo profissional não fique cuidando desses pacientes.

Na hora da retirada de medicação eu sempre chamo a atenção das meninas para colocar nome, leito, separar quando tem o mesmo nome - principalmente aqui em cima que é enfermaria - quando possui o mesmo nome, como Maria, João, tentar separar de escala de técnico que fica responsável. (E2)

No momento de administrar os medicamentos, há a conferência com a identificação do paciente e a verificação se o paciente possui alguma alergia medicamentosa. Nestes casos, é colocada a pulseira sinalizadora de alergia no paciente, assim como descrito no quadro do posto de enfermagem e na prescrição médica.

Alguns enfermeiros referiram a importância de informar aos pacientes quais medicamentos serão administrados, assim como checar se já receberem tais medicamentos antes, no intuito de estimular a participação do paciente e dos familiares no cuidado.

Eu acho importante a gente orientar o paciente a confirmar sempre com a gente [...] eu falei para o paciente a medicação que eu ia fazer daí o paciente falou 'Olha, não lembro de ter recebido essa medicação antes'! Dai, quando eu fui olhar não era dele, daí não fiz. Dei graças a Deus que ele me falou, poupei um erro. (EA6)

Um dos principais eventos adversos relatados pelos enfermeiros é o risco de queda. Neste sentido, na subcategoria "Identificação e cuidados ao paciente com risco de queda", os enfermeiros apresentam o perfil predominante dos pacientes com este risco na unidade: idosos, aqueles com dificuldade de marcha, em pós-operatório, com maior complexidade assistencial (dependentes dos cuidados de enfermagem), assim como aqueles pacientes que utilizam ansiolíticos.

Para identificar o paciente com risco de queda, os enfermeiros utilizam a pulseira de risco de queda e disponibilizam um termo de ciência sobre o evento ao paciente e familiar. Nesse termo constam os cuidados que o paciente e familiares/acompanhantes devem ter para prevenção de queda.

Os enfermeiros realizam vários cuidados para prevenção de queda, como: orientação ao paciente e familiar, no intuito de empoderá-los e participarem do cuidado; paciente estar sempre acompanhado de algum familiar ou cuidador; orientação à equipe de enfermagem; manter grades elevadas, campainha próxima ao paciente e cama com altura próxima ao chão; evitar ausência dos profissionais durante os procedimentos; manter os corredores ou áreas próximas ao paciente livre de objetos que possam dificultar a deambulação; cuidados durante o transporte desse paciente; durante o banho, manter sempre próximo uma cadeira de banho; avaliação diária do nível de consciência dos pacientes, para identificar confusão, delirium ou agitação, conforme expresso na fala:

O paciente em pós-cirúrgico, ou paciente idoso ou algum paciente com algum problema de marcha. Então, o simples fato de você falar, deixar a campainha do lado, deixar as grades elevadas, sempre estar ao lado dele, sempre orientar a chamar a equipe de enfermagem. (EA4) 
Na subcategoria “Cuidados ao paciente com risco de desenvolver úlcera por pressão", primeiramente é necessário identificar os pacientes que apresentam esse risco. Para os enfermeiros pesquisados, os pacientes idosos, acamados, dependentes, com uso de fralda ou sonda possuem maior risco de desenvolver úlcera por pressão.

Após a identificação destes pacientes, iniciam-se os cuidados de prevenção, com a mudança de decúbito a cada 2 horas, utilização do colchão piramidal (colchão perfilado), massagem e hidratação da pele e orientações à equipe de enfermagem. Caso o paciente já apresente alguma úlcera, o enfermeiro avalia a lesão, indica e inicia o tratamento.

\section{Melhores práticas de liderança do enfermeiro}

Essa categoria é composta por cinco subcategorias relacionadas à liderança dos enfermeiros para garantir um cuidado seguro.

A primeira subcategoria, "Assistência integral ao paciente", afirma que o enfermeiro além de ser líder de uma equipe e realizar a gestão da unidade, é responsável também por prestar cuidado direto ao paciente. Para os enfermeiros, assistência integral ao paciente, seja aquela realizada somente pelo enfermeiro ou pelos membros da equipe de enfermagem, é considerada como melhor prática no cuidado seguro. Através da assistência integral, é possível conhecer o paciente por completo, não somente os aspectos físicos, identificando suas potencialidades e necessidades de cuidado.

Aqui a gente presta uma assistência integral, nós enfermeiros [...] Então, eu gosto de estar presente ali, de estar vendo o que está acontecendo. Porque eu sei do meu paciente, o que ele está precisando, da maneira de cuidar dele [...] para que evite esses eventos adversos. (EA3)

Na subcategoria "Comunicação como prática segura de cuidado" evidenciou-se a importância da comunicação entre as equipes para a prevenção de eventos adversos. Salientam o registro dos cuidados na evolução e a passagem de plantão de forma verbal e escrita, já que há na instituição um protocolo para passagem de plantão.

Diante da importância dos registros, a "Sistematização da Assistência de Enfermagem (SAE)" foi considerada como uma prática relevante para garantir o cuidado seguro ao paciente. Inicia-se com o histórico de enfermagem, onde é possível identificar os riscos e as necessidades de cuidados, seguido pela realização de um plano de cuidado. Diariamente os enfermeiros preenchem as escalas assistenciais, como Braden, Stratify, Escala Assistencial de Complexidade e de Flebite, as quais auxiliam os enfermeiros a avaliar o dimensionamento de pessoal, o grau de risco dos pacientes, assim como a avaliação das intervenções a partir da evolução e prescrição de enfermagem, possibilitando a gestão do cuidado.

"As relações de trabalho na equipe" foram evidenciadas como uma facilidade para reduzir os eventos adversos e garantir um cuidado seguro, através da comunicação, troca de experiência, ajuda mútua, cooperação e bom relacionamento entre os membros da equipe.

A subcategoria "Orientação como prática de cuidado seguro" está relacionada à orientação que deve ser realizada junto aos pacientes, familiares e equipe de enfermagem. Além de se configurar como uma ação de liderança do enfermeiro, garante também a resolutividade das dúvidas, norteia o cuidado da equipe de enfermagem, empodera o familiar e paciente, e estimula a participação no cuidado, reduzindo os eventos adversos e garantindo um cuidado seguro.

Ah, orientação [...] passar sempre para o seu colega o que está acontecendo com o seu paciente, caso suspeita de alguma coisa que possa vir a acontecer já orientá-lo também, orientar o acompanhante [...] tudo o que possa prevenir, para algum procedimento que você for fazer, faça bem feito, oriente, enfim, para poder prevenir que aconteça alguma coisa, a prevenção. (EA4)

\section{Proposições para o aprimoramento das melhores práticas}

Os enfermeiros elencaram que existem possibilidades e necessidades no aprimoramento das 
melhores práticas para o cuidado seguro à pessoa com doença cardiovascular, apresentados através de sete subcategorias.

$\mathrm{Na}$ "Administração segura de medicamentos", os enfermeiros elencaram a necessidade de implementar a dupla checagem dos medicamentos também no momento da administração ao paciente, assim como qualificar o registro dos profissionais após a administração do medicamento e justificar os casos que o paciente não o aceitou.

Na subcategoria "Capacitação dos profissionais", foi sustentado que através da qualificação e a atualização dos conhecimentos, os profissionais se sentem mais valorizados e motivados, exercendo um cuidado com mais segurança. A presença da educação permanente nos setores é considerada uma melhor prática para alcançar a redução dos eventos adversos. A participação do enfermeiro nessa prática é essencial para identificar as necessidades de treinamentos e organizar esses momentos com a sua equipe.

Na subcategoria "Dimensionamento adequado de pessoas e retenção de profissionais", verificouse que para os enfermeiros há a necessidade de desenvolver estratégias para diminuir a rotatividade e rever o dimensionamento dos profissionais. A alta rotatividade (tanto de técnicos de enfermagem quanto de enfermeiros) e o dimensionamento inadequado das equipes acarretam em sobrecarga do trabalho, com risco do cuidado ser realizado rapidamente e com pouca atenção, podendo ocasionar ações inseguras e eventos adversos.

Eu acho que a dificuldade nossa é a rotatividade [...] Perde-se totalmente a segurança do paciente, é como se a gente colocasse o paciente numa linha de tiro, porque é bem difícil! [...] Quando está começando alguém novo, é treinar, capacitar, mas corre um grande risco né? (E3)

Na subcategoria "Informação sobre segurança do paciente e notificações dos eventos adversos", verificou-se que a equipe de enfermagem não está familiarizada sobre o termo segurança do paciente e evento adverso, qual a importância das notificações desses eventos e a não penalização dos erros. Para os enfermeiros, através da conscientização é possível intensificar as ações de prevenção de eventos adversos.

Então, acho que é uma questão de treinamento com a equipe de enfermagem, com os enfermeiros e os técnicos. Porque se você for perguntar para eles, eles não sabem o que é evento e o que deve ser notificado. (E2)

Através da subcategoria a "Disponibilidade de materiais e espaço adequado para realizar o cuidado seguro", os enfermeiros referiram à falta de materiais ou em manutenção, principalmente para os pacientes em isolamento de contato, e espaço inadequado para realizar os cuidados como itens que podem dificultar o cuidado e ocasionar eventos adversos aos pacientes.

Na subcategoria "Cultivar a comunicação entre as equipes e evoluir com o uso do protocolo de passagem de plantão", verificou-se a necessidade de desenvolver a comunicação entre os profissionais, já que esta é uma melhor prática identificada pelos enfermeiros. Através da comunicação e da troca de ideias, é possível aprender com as experiências e melhorar o cuidado prestado.

"Aperfeiçoar as relações com os acompanhantes de forma institucional" refere-se à presença do acompanhante durante a internação do paciente. Na instituição, todos os pacientes podem ter acompanhantes nas 24 horas e o horário de visita nas unidades de internação é estendido, ocorrendo ao longo do dia. Para os enfermeiros há certa dificuldade em lidar com essas práticas, por preocupação referente à prevenção de infecção hospitalar e devido ao fato de muitos acompanhantes serem idosos, os quais possuem fragilidades emocionais e de saúde, podendo dificultar a sua inclusão nos cuidados do paciente.

\section{DISCUSSÃO}

As melhores práticas elencadas neste estudo corroboram com as "Seis Metas Internacionais de Segurança do Paciente" da Organização Mundial da Saúde e com os protocolos básicos definidos pela portaria n $529 / 2013$ do Ministério da Saúde ${ }^{(8)}$. 
A identificação do paciente é a primeira meta internacional de segurança, considerada um dos principais fatores para monitorar a segurança do paciente, especialmente por agir como protetor de eventos adversos ${ }^{(9)}$. Além da pulseira, a instituição utiliza também a identificação no leito como mais uma prática de prevenção ao evento adverso.

Para a administração segura dos medicamentos, identificou-se como necessidade de aprimoramento a implementação da dupla checagem e qualificação do registro profissional após a administração das medicações. Durante a internação hospitalar, os pacientes com doenças cardiovasculares utilizam diariamente os medicamentos de alta vigilância, os quais possuem risco maior de provocar danos significativos aos pacientes caso ocorra falha no processo de utilização ${ }^{(10)}$; por isso a importância de utilizar a dupla checagem na dispensação, preparo e administração dos medicamentos de alta vigilância, bem como seu registro adequado, que podem potencializar o monitoramento sobre o paciente e avaliação terapêutica.

A equipe de enfermagem tem um papel importante na identificação dos fatores de risco dos pacientes e do ambiente, os quais contribuem para a prevenção de eventos adversos, como a queda e úlcera por pressão. O perfil dos pacientes com risco de queda abordados neste estudo está em consonância com a literatura ${ }^{(5,11)}$.

Devido a este perfil, é importante reforçar a necessidade da reavaliação diária do risco de desenvolvimento da úlcera por pressão, já que esta prática permite aos profissionais de saúde ajustar sua estratégia de prevenção conforme as necessidades do paciente. A participação da equipe multiprofissional junto à equipe de enfermagem na prevenção da úlcera de pressão é fundamental, já que poderão ser necessários ajustes nutricionais, intervenções para auxiliar a mobilização ou mobilidade dos pacientes, entre outras medidas ${ }^{(12)}$.

A prática do cuidado sistematizado a pacientes submetidos à cirurgia cardíaca, além de melhorar a qualidade da assistência, contribui para o reconhecimento da importância das ações de enfermagem em qualquer nível de assistência ${ }^{(13)}$. A SAE vem sendo implementada na prática assistencial, e confere maior segurança aos pacientes, melhora a qualidade da assistência e a autonomia aos profissionais de enfermagem, por organizar o trabalho quanto ao método, número de pessoas e adequação de instrumentos, além de viabilizar a operacionalização do Processo de Enfermagem ${ }^{(14)}$. Neste contexto, a utilização da SAE garante um cuidado humano, individualizado e centrado nas necessidades humanas básicas, além de garantir um plano de cuidado ao paciente e nortear as ações do enfermeiro e equipe de enfermagem.

O enfermeiro, ao gerenciar o processo de cuidado e no exercício da gerência da unidade, como líder da equipe de enfermagem, está inserido no ponto central do processo de capacitação e desenvolvimento da sua equipe, devendo ficar atento às necessidades de treinamento, bem como implementando estratégias de ensino-aprendizagem ${ }^{(15)}$. Assim, a presença da educação permanente é considerada pelos participantes como uma melhor prática no intuito de desenvolver conhecimentos, habilidades e práticas, possibilitando um cuidado qualificado e seguro ao paciente. A orientação e o treinamento de pessoal são considerados importantes não apenas na socialização e no processo de formação, como também para garantir o clima motivacional da equipe e partilhar responsabilidades de forma integrada ${ }^{(16)}$.

Neste sentido, ressalta-se a importância da comunicação como instrumento de liderança do enfermeiro para o cuidado seguro. A passagem de plantão implica em um processo de comunicação entre os profissionais, assim como a transferência de responsabilidade do cuidado. A complexidade do tipo de informação transmitida, os meios de comunicação adotados e as características dos profissionais da equipe tem impacto na efetividade e eficiência da passagem das informações e consequentemente na segurança do paciente ${ }^{(17)}$.

Além da comunicação entre os membros da equipe, é importante considerar a comunicação entre profissionais e os pacientes/acompanhantes. Esta deverá desenvolver-se de forma adaptada ao paciente e sua situação, ou seja, o enfermeiro deverá considerar as especificidades culturais e linguísticas do paciente, bem como o seu grau de desenvolvimento cognitivo ${ }^{(15)}$. Diante deste contexto, os enfermeiros precisam valorizar a comunicação com o paciente e acompanhante, buscando viabilizar sua participação ativa no cuidado e consolidar-se como referência para o cuidado. 
Outro fator importante para garantir o cuidado seguro é o dimensionamento adequado da equipe de enfermagem, já que a manutenção de número reduzido de enfermeiros pode comprometer a supervisão do serviço, a qualidade do cuidado e igualmente coloca em risco a segurança do paciente ${ }^{(18)}$. Revisão integrativa de literatura identificou que há relação entre o quantitativo de enfermagem subestimado e o aumento das taxas de infecções, mortalidade, quedas, pneumonia associada à ventilação mecânica, extubação acidental e tempo de internação(19).

As instituições que apresentam dimensionamento adequado de enfermagem, que se preocupam com a capacitação e a retenção de enfermeiros, permitem uma assistência de enfermagem com foco na qualidade, favorecendo a prevenção e detecção precoce de possíveis eventos adversos ${ }^{(20)}$.

Por fim, deve-se evoluir com a cultura de segurança na Instituição, já que uma cultura de segurança positiva está associada à adoção de comportamentos seguros e diminuição de eventos adversos, diretamente relacionados à segurança do paciente ${ }^{(21)}$. Neste contexto, verificou-se a necessidade de sensibilizar a equipe de enfermagem sobre a temática segurança do paciente, em utilizar a ocorrência dos eventos adversos como uma oportunidade de orientação e assim, conseguir envolvê-los na importância das notificações e nas melhores práticas de prevenção e cuidado seguro.

\section{- CONCLUSÃO}

As práticas apresentadas estão em consonância com os protocolos do Ministério da Saúde e demais literaturas, consolidando-se como práticas relevantes para o cuidado seguro, atuais e adequadas à realidade estudada.

Embora os achados deste estudo apontem a realidade de um hospital privado, foram evidenciadas melhores práticas no cuidado direto ao paciente, nas ações de liderança do enfermeiro e práticas que precisam ser aperfeiçoadas, que poderão servir de subsídio para profissionais de contextos similares ou orientar gestores públicos na adoção de instrumentos e recursos já utilizados em instituições privadas visando à segurança do paciente.

A cultura da segurança deve ser internalizada pelos profissionais, sendo necessário ampliar as estratégias de educação permanente sobre o tema, melhorar os processos e condições de trabalho, adequar o dimensionamento dos profissionais e entender a presença dos familiares/acompanhantes como coparticipantes do cuidado seguro.

Embora o estudo tenha contemplado os enfermeiros que atendiam aos critérios de inclusão, destaca-se como limitação não considerar o depoimento dos técnicos de enfermagem, uma vez que realizam atividades diretas de cuidado com os pacientes.

\section{- REFERÊNCIAS}

1. Springhouse. As Melhores práticas de enfermagem: procedimentos baseados em evidências. 2a. ed. Porto Alegre: Artmed; 2010.

2. University Of Iowa. College of Nursing [Internet]. Iowa: University Of lowa. Csomay Center - Best Practices for Healthcare Professionals; 2014 [acesso em 28 set 2014]. Disponível:

http://www.nursing.uiowa.edu/hartford/best-practices-for-healthcare-professionals

3. Costa VT, Meirelles BHS, Erdmann AL. Melhores práticas do enfermeiro gestor no gerenciamento de risco. Rev. Latino-Am. Enfermagem [Internet] 2013; 21(5) [acesso em 01 mar 2016]. Disponível:

http://www.scielo.br/pdf/rlae/v21n5/pt_0104-1169-rlae-21-05-1165.pdf

4. Agência Nacional de Vigilância Sanitária (ANVISA). Gerência de Vigilância e Monitoramento em Serviços de Saúde. Gerência Geral de Tecnologia em Serviços de Saúde. Assistência Segura: Uma Reflexão Teórica Aplicada à Prática. Brasília: ANVISA; 2013.

5. Teixeira TCA, Cassiani SHB. Análise de causa raiz de acidentes por quedas e erros de medicação em hospital. Acta paul. enferm. [Internet] 2014; 27(2) [acesso em 05 jan 2016]. Disponível: 
6. Novello MF, Mesquita ET, Rivas M, Lanzieri PG, Jorge BAL, Motta JMT, et al. Qualidade e segurança assistencial aplicada à cardiologia: as contribuições da experiência americana. Rev Bras Cardiol. [Internet] 2011; 24(3) [acesso em 05 jan 2016]. Disponível:

http://sociedades.cardiol.br/socerj/revista/2011_03/a_2011_v24_n03_05qualidade.pdf

7. Bardin L. Análise de conteúdo. 4a. ed. Lisboa: Edições 70; 2009.

8. Ministério da Saúde (BR). Portaria SAS/MS 529, de $1^{\circ}$ de abril de 2013. Institui o Programa Nacional de Segurança do Paciente (PNSP). Brasília; 2013.

9. Neves LAC, Melgaço RMT. A identificação do paciente como indicador de qualidade [monografia]. Rio de Janeiro (RJ): Universidade Federal do Estado do Rio de Janeiro; 2011.

10. Instituto para Práticas Seguras no uso de Medicamentos (ISMP). Medicamentos potencialmente perigosos. [Internet] 2014 [acesso em 17 set 2014]. Disponível: http://www.ismp-brasil.org/site/index/medicamentos/

11. Ministério da Saúde (BR), Agência Nacional de Vigilância Sanitária (ANVISA), Fundação Oswaldo Cruz (FIOCRUZ). Anexo 01: Protocolo prevenção de quedas. Brasília; 2013.

12. Borghardt AT, Prado TN, Araújo TM, Rogenski NMB, Bringuente MEO. Avaliação das escalas de risco para úlcera por pressão em pacientes críticos: uma coorte prospectiva. Rev. Latino-Am. Enfermagem [Internet] 2015; 23(1) [acesso em 07 jan 2016]. Disponível: http://dx.doi.org/10.1590/0104-1169.0144.2521

13. Carvalho ML, da Silva MHR, Carvalho ML, Elias CMV, da Silva KR, dos Santos MC. Assistência de enfermagem na UTI a pacientes submetidos à cirurgia cardíaca. R. Interd. [Internet] 2013; 6(4) [acesso em 10 jan 2016]. Disponível: http://revistainterdisciplinar.uninovafapi.edu.br/index.php/revinter/article/view/195/pdf_68

14. Tannure MC, Pinheiro AM. SAE - Sistematização da assistência de enfermagem - guia prático. São Paulo: Guanabara Koogan; 2011.

15. Santos APA. O enfermeiro no pós-operatório de cirurgia cardíaca: competências profissionais e estratégias da organização [dissertação]. Ribeirão Preto (SP): Universidade do Estado de São Paulo; 2015.

16. Duarte SCM, Stipp MAC, Silva MM, Oliveira FT. Eventos adversos e segurança na assistência de enfermagem. Rev. Bras. Enferm. [Internet] 2015; 68(1) [acesso em 11 jan 2016]. Disponível:

http://dx.doi.org/10.1590/0034-7167.2015680120p

17. Santos MC, Grilo A, Andrade G, Guimarães T, Gomes A. Comunicação em saúde e a segurança do doente: problemas e desafios. Rev Port Saúde Pública [Internet] 2010; Vol Temat(10) [acesso 11 jan 2016]. Disponível: http://proqualis.net/artigo/comunica $\%$ C3\%A7\%C3\%A3o-em-sa\%C3\%BAde-e-seguran $\%$ C3\%A7a-do-doenteproblemas-e-desafios

18. Lorenzini E, Deckmann LR, Costa TC, Silva EF. Dimensioning of nursing staff: an integrative review. Ciênc Cuid Saúde [Internet] 2014; 13(1) [acesso em 12 jan 2016]. Disponível:

https://dx.doi.org/10.4025/cienccuidsaude.v13i1.15959

19. Versa GLGS, Inoue KG, Nicola AL, Matsuda LM. Influência do dimensionamento da equipe de enfermagem na qualidade do cuidado ao paciente crítico. Texto contexto-enferm. [Internet] 2011; 20(4) [acesso 12 jan 2016]. Disponível: http://dx.doi.org/10.1590/S0104-07072011000400020

20. Oliveira RM, Leitao IMTA, Aguiar LL, Oliveira ACS, Gazos DM, da Silva LMS, et al. Evaluating the intervening factors in patient safety: focusing on hospital nursing staff. Rev Esc Enferm USP [Internet] 2015; 49(1) [acesso 13 jan 2016]. Disponível: https://dx.doi.org/10.1590/S0080-623420150000100014

21. de Souza VS, Kawamoto AM, de Oliveira JLC, Tonini NS, Fernandes LM, Nicola AL. Erros e eventos adversos: a interface com a cultura de segurança dos profissionais de saúde. Cogitare enferm. [Internet] 2015; 20(3) [acesso em 01 mar 2016]. Disponível: http://dx.doi.org/10.5380/ce.v20i3.40687 